\title{
LA ESCRIBANÍA DE LA SALA DEL CONSELL: UN OFICIO CONTROVERTIDO
}

\author{
Marta DÍEZ SÁNCHEZ \\ Universidad de Alicante
}

\begin{abstract}
Resumen
El artículo recoge las dificultades que rodearon la provisión de la escribanía de la Sala del Consell de la ciudad de Alicante en el período comprendido entre 1665 y 1680 , con la sucesión de hasta cinco escribanos en el oficio, dos de los cuales fueron encausados por diferentes irregularidades. Tras los motivos que llevaron al procesamiento de los escribanos Joseph Arcayna y Victoriano Tredós subyacen las luchas por el poder entre las distintas facciones de la oligarquía local.
\end{abstract}

\begin{abstract}
This article points out how difficult was to obtain the provision of the «escribanía» in the Townhall of Alicante during the years 1665 till 1680, with the succession of up to five town clerks in the job, two of those were prosecuted by different irregularities. The power struggles between the different factions of the local oligarchy underlie the reasons that carried on to the processing of the town clerks Joseph Arcayna and Victoriano Tredós.
\end{abstract}

En este artículo queremos tratar un aspecto de la administración alicantina que nos llamó la atención cuando, tiempo atrás, nos ocupábamos en el estudio de la organización municipal y de la gestión de su tesorería durante la segunda mitad del siglo XVII'. Si bien los hechos que vamos a recoger aquí corroboran y amplían el apoyo documental sobre el que basamos nuestras conclusiones acerca de los defectos del sistema administrativo, principalmente en las que se refieren a la supeditación del in-

1. Este tema fue tratado en nuestra Memoria de Licenciatura Aproximación al estudio de las administraciones locales en época foral: organización y gestión económica de la hacienda municipal de Alicante en la segunda mitad del Seiscientos, presentada en la Facultad de Filosofía y Letras de la Universidad de Alicante el 27 de junio de 1997 y que próximamente será publicada por el Instituto de Cultura «Juan Gil-Albert» en su colección Textos Universitaris bajo el título La Hacienda nunicipal de Alicante en la segunda mitad del siglo XVII. Dado que en las fechas de redacción de este artículo el libro aún se encuentra en prensa, las referencias que hagamos a su contenido corresponderán a las páginas de la Memoria. 
terés público al interés particular de quienes monopolizaban los oficios municipales, decidimos no tratarlo entonces por considerar que entrar al detalle en la cuestión no encajaba en la estructura del trabajo que previamente habíamos preparado.

El caso podría plantearse así: en los quince años que median entre 1665 y 1680 , la escribanía del Consell de la ciudad de Alicante, oficio que, a diferencia del resto de los cargos de la administración municipal, no estaba sujeto a elección o remoción temporal, experimentó la sucesión de hasta cinco titulares, y no precisamente por fallecimiento ni por renuncia del titular; circunstancia esta última que, por otra parte, se nos antoja impensable, dadas las infinitas posibilidades que el puesto ofrecía a individuos con un mínimo de pericia, grandes dosis de ambición y, por supuesto, carentes de escrúpulos, para medrar social y económicamente en el escalafón ciudadano. La situación se desarrolla en un ambiente ciertamente viciado; incluso podría decirse que es una consecuencia del mismo. Las dificultades que atraviesa la provisión de la escribanía dejan entrever las luchas entre las distintas facciones de la oligarquía alicantina por un mayor control del gobierno local, entendiendo siempre éste como una mayor participación en los fraudes económicos a la tesorería de la ciudad: distracción de ingresos, participación en arrendamientos de abastos municipales y otras rentas -hecho especialmente grave porque estaba prohibido expresamente por los estatutos de gobierno-, utilización del dinero público para transacciones particulares, falseamiento de la contabilidad, etc. Estas dificultades nos permiten, además, inferir otro tipo de conclusiones como, por ejemplo, el alto precio que pagaba la administración real a cambio de la fidelidad política de las élites ciudadanas, permitiendo que unos cuantos disfrutaran de unas rentas que ésta dejaba de percibir, aun necesitándolas con apremio, y su incapacidad manifiesta por arbitrar soluciones legales y administrativas que pudieran extirpar esta lacra.

En este contexto, que en absoluto es excepcional, y cuyas consecuencias, en el caso particular de Alicante, posiblemente revistieron menor gravedad que en otros municipios debido a las relativa buena salud de sus rentas ${ }^{2}$, la provisión de la escribanía resultaba una cuestión de especial importancia ${ }^{3}$. Con un individuo afín al frente de la escribanía, entre cuyas competencias figuraba el registro de todas las anotaciones contables, el «equipo» formado por jurados y escribano se aseguraba una forma rápida de obtener pingües beneficios:

"La clavería comuin tiene el cargo de recibo y cargos de la Cindad y se halla que el clavario viene a ser oy, una testaferrea, pues en su poder no entra un real, o muy poco,

2. Aproximadamente el $80 \%$ de las entradas en la tesorería procedían de impuestos que repercutían sobre el tráfico y el volumen comercial de su puerto. La intensa actividad desarrollada en él permitía a la ciudad mantener una situación financiera bastante saneada, aunque en ocasiones los condicionantes de la política exterior de la Monarquía provocaran crisis episódicas. Vid. DÍEZ SÁNCHEZ, M.: Aproximacion al estudio de las administraciones locales..., pp. 172-192.

3. Agustín Rubio. en su obra dedicada a la escribanía municipal de la ciudad de Valencia en la Baja Edad Media, alude precisamente a esta cuestión y nos muestra las controversias suscitadas durante el siglo $X V$ entre la ciudad y las autoridades reales por el nombramiento de este oficial. RUBIO VELA, A., L'escrivania municipal de València als segles XIV i XV: burocràcia, política i cultura, Valencia, 1995 , pp. 53-85. 
quedándose el escrivano y los jurados lo que sobra después de pagados los censos y cargos de la Ciudad...» ${ }^{4}$

En las páginas siguientes vamos a referirnos concretamente a las actuaciones de Joseph Arcayna y Victoriano Tredós al frente de la escribanía del Consell. Los años en los que ejercieron sus oficios se corresponden con un período especialmente delicado para la administración municipal, intervenida desde 1662 por oficiales reales a raíz de varias denuncias que hacían públicas las irregularidades cometidas por la oligarquía y que también salpicaron a los citados oficiales, hasta el punto de ser objeto de sendos procesos judiciales. Pero antes de entrar de lleno en la materia, creemos conveniente hacer unas breves consideraciones sobre la forma de provisión y las competencias de este oficio.

La escribanía de la Sala del Consell de la Ciudad era un cargo de carácter vitalicio. Hasta mediados del siglo XVII, la facultad de nombrar a un nuevo escribano, por renuncia o muerte del titular, había correspondido al rey. Sin embargo, en las Cortes celebradas en Valencia en 1645 Felipe IV accedió a atender la petición de algunas ciudades y villas, entre ellas Alicante ${ }^{5}$, de disponer de autoridad para elegir y nombrar al candidato. En el caso concreto de nuestra ciudad, esta facultad se entendería en vigor «després dels dies del primer sucesor del que huy la posehirà y fins a les primeres corts» ${ }^{\circ}$.

La principal ocupación del escribano era la redacción y certificación de todos los documentos y registros emanados de la autoridad local. Dentro de sus funciones entraba, por lo tanto, la formalización y actualización de todos los libros contables de la tesorería así como de cualquier otra administración o arrendamiento. Dada la naturaleza de sus competencias, estaba obligado a acudir a todos los actos oficiales y se requería su asistencia a las dependencias del Consell de la ciudad por un mínimo de cuatro horas diarias. Sus funciones estaban remuneradas con un salario de 95 libras anuales, sin contar con los derechos que percibía por la certificaciones y copias de documentos oficiales, a las que hay que añadir 10 libras más para gastos de su despacho y otras tantas por encargarse del denominado libro de la giradora, en el que se anotaban los turnos de riego de la huerta alicantina ${ }^{7}$.

4. Archivo de la Corona de Aragón (en adelante, A.C.A.), Sección Secretaría de Valencia (en adelante, S.V.), Leg. 679, Doc. 19. Memorial de D. Diego Sanz de La Llosa dirigido al rey en 1662.

5. Las otras poblaciones fueron Xàtiva, Ontinyent, Llíria, Olleria, Mutxamel y Agullent, si bien a las dos últimas no les fue aceptada la petición. Vid. GUIA MARÍN, Ll., Cortes del reinado de Felipe IV. II. Cortes valencianas de 1645, Valencia, 1984, p. 115., n. 78.

6. Ibídem, p. 302.

7. La documentación que quedaba a cargo del escribano abarcaba una gran lista, como muestran los estatutos de gobierno de 1669: «ha de recibir francamente los autos y escrituras que otorga la Ciudad, como son ordinaciones de Consejo, Sindicados para qualquier efeto que sean, nominaciones, extracciones, llevar libro Judiciario de Sitiadas, de Albaranes, de guardas y, amas, libro de censales, como libro de caxa de Mercaderes, poniendo en contra las pagas; haziendo mención de las ápocas, memorias de obras, de municiones, libro de registro de mandatos (...), y el espillet del Clavario, y administración, copiar y registrar en sa lugar privilegios, letras y provisiones Reales, y llevar libro dellos, y libro de las cartas misivas que la Ciudad escribe, y otro de las que recibe (...); lievar libro particular de todo lo concerniente al morbo, llevar otro libro de ápocas de la Clavería, y otro de la Matrícula de los inseculados, otro de la hazienda y títulos della, con que los insecularen, y asistir en todos los acompañamientos y actos públicos a la Ciudad". ALBEROLA ROMA, A. y PATERNINA BONO, M"J., Ordenanzas municipales. Alicante, 1459-1669, Alicante, 1989, pp. 383-387. 
En la fecha en la que tuvieron lugar las últimas Cortes del Reino de Valencia, ocupaba la escribanía municipal de Alicante Ginés Arcayna. Su nombramiento se había producido el 6 de agosto de 1634, por privilegio real. Aproximadamente seis meses después de la clausura de las Cortes, un nuevo privilegio, datado en la corte el 7 de junio de 1646, autorizaba a Arcayna a elegir a su sucesor. El escribano hizo uso de esta merced, en favor de su hijo Joseph, en su último testamento, redactado el 23 de octubre de $1653^{\circ}$.

Cuando le sobrevino la muerte a su padre, Joseph tenía 17 años, razón por la cual el virrey, duque de Montalto, designó a Diego Pastor como escribano con carácter provisional. Una vez en el puesto y, al parecer, con el apoyo de los jurados de la ciudad, Pastor solicitó directamente al rey que se le mantuviese al frente de la escribanía durante los ocho años que faltaban para que Arcayna alcanzase la mayoría de edad. El virrey, molesto por la licencia que se tomó el escribano en funciones sin hacerle partícipe a él, se manifestó en contra de tal petición y, en cambio, se mostró partidario de incorporar pronto a su oficio al joven Arcayna, a quien consideraba persona experta y preparada por haber trabajado como ayudante de su padre. La iniciativa de Pastor acabaría provocando su sustitución en 1654 por el notario Juan Bautista Boyer, quien debería compartir con Joseph Arcayna el salario y demás emolumentos correspondientes a su oficio".

Cuando, alcanzada la mayoría de edad, ocupó finalmente su puesto, Arcayna no tardó en dar muestras de su pericia, aunque no precisamente en la medida de las cualidades alabadas por el virrey. En un memorial dirigido a Felipe IV en 1662, D. Diego Sanz de La Llosa, gobernador de Orihuela, no escatimaba palabras para calificar la actuación de Joseph, a quien consideraba «el sangano desta República y primer móbil de todos los daños, por tener el dinero en su poder» ${ }^{11}$. Las acusaciones de Sanz de La Llosa presentan a Arcayna como el prototipo del munícipe corrupto: obstruía deliberadamente las sesiones del Consell", manipulaba la práctica contable, formalizaba arbitrariamente las tramitaciones de las diligencias de cobro, demorando las que afectaban a sus allegados, y transgredía gravemente los estatutos de gobierno, teniendo parte -eso sí, bajo testaferros- en casi todos los arrendamientos de abastos y sisas municipales ${ }^{12}$. Nos consta, según un documento anónimo datado en años posteriores, que durante su ejercicio llegó a tener la cuarta parte del contrato de arrendamiento del derecho de saladura y tabaco, a través de su hermano Jaime. Dicho arrendamiento, del que no hemos podido averiguar su fecha, se saldó con pérdidas, pero, según la misma fuente, Arcayna se libró de responder de las 1.000 libras que le correspondía abonar a

\footnotetext{
8. A.C.A., S.V., Leg. 805, Doc. 40/3.

9. A.C.A., S.V., Leg. 900 , Doc. $40 / 1$

10. A.C.A., S.V., Leg. 679, Doc. 19/1. Vid. transcripción en DÍEZ SÁNCHEZ, M., Aproximación al estudio de las administraciones locales en época foral..., pp. 318-324.

11. «Las ordinasiones consiliares se sospecha que no se alargan por el escrivano y, quando se alarguen, ay omisiones muy culpables y pernisiosas. Fúndase la sospecha en la dificultad y repugnansia que ay para no dallas quando se piden, y se a advertido que quando algún consejero contradice o protesta al votar, se repara en alargar su protesto y disentimiento $y$, tal vez, no se halla la formalidad del voto...». Ibídem.

12. A.C.A., S.V., Leg. 679 , Doc. 24.
} 
las arcas locales gracias a la complicidad de su primo Pedro Maltés, que ejercía como abogado del Consell municipal, y de otros oficiales como el subsíndico ${ }^{13}$.

En 1663, un año después de la llegada a la Corte del memorial remitido por el gobernador, y como consecuencia de la visita de residencia encomendada por el monarca al Dr. D. Braulio Esteve para poner freno a los desmanes y abusos que se estaban produciendo en el gobierno municipal ${ }^{14}$, Arcayna fue suspendido en sus funciones por el visitador. Se le abrió proceso, como a la mayoría de los oficiales municipales en ejercicio, bajo la acusación de transgredir los estatutos de gobierno y de apropiación fraudulenta de fondos ${ }^{15}$. Concluida la causa al año siguiente, fue hallado culpable de las acusaciones formuladas en su contra y, acto seguido, fue destituido definitivamente del cargo y condenado a pena de privación de oficio público y a una multa de 2.000 libras que, en caso de no ser satisfecha, comportaría el destierro en Orán.

Para hacer frente a la sanción económica, de la cual 785 libras correspondían al tribunal de la visita, Arcayna vendió a carta de gracia una propiedad valorada en 1.740 libras a Pedro Juan Domenech. El contrato estipulaba que Arcayna recibiría 800 libras en el momento de la compra y el resto a pagar, con los correspondientes intereses, en cuatro plazos. Pronto surgirían los problemas, ya que la casa ahora vendida había sido utilizada por Arcayna con anterioridad como garantía para la obtención de ciertas cantidades mediante censos. Cuando comenzaron a vencer los plazos de las pensiones de estos censos, que importaban en conjunto una cantidad superior a las 1.200 libras, el visitador Esteve ordenó al nuevo propietario destinar los pagos que le quedaban pendientes a satisfacer a los censalistas, entre los que se encontraban el convento de monjas clarisas de Santa Faz y la iglesia de Santa María. Esta obligación de dar prioridad a los censalistas, privó a Arcayna de la posibilidad de reunir el dinero necesario para eludir el destierro a la plaza de Africa, pena que, al parecer, fue finalmente ejecutada ${ }^{16}$. El escribano, sin embargo, no se amilanó ante la gravedad de la situación y apeló la sentencia del tribunal de la visita ante el Consejo de Aragón.

Juán Bautista Boyer, que había venido ejerciendo como escribano en funciones tras la suspensión inicial de Arcayna, solicitó su ratificación en el mismo puesto, una vez que el tribunal de la visita había apartado a éste del cargo. Requerida por Felipe IV al marqués de Astorga, virrey de Valencia, la redacción de una terna para proveer el cargo hasta que se decidiese la apelación de Arcayna, éste demostró sus preferencias por el Dr. D. Victoriano Tredós y Pascual, en primer lugar, seguido del notario Juan Bautista Boyer y, en último lugar, de Honorato Gosálbez. En relación a Boyer manifestó que, si bien le tenía como «sugeto ábil y inteligente», «aunque fue nombrado en el exerçicio mientras la residencia, no se aplicó a este cuidado y ocupación sino por Honorato Boyer, su sobrino muy confidente del que quedo privado, y que si llegase a obtenerle en propiedad [el oficio de escribano] sería de inconveniente el

13. A.C.A, S.V., Leg. 811 , Doc. $40 / 39$.

14. Sobre la visita del Dr. Esteve véase DÍEZ SÁNCHEZ, M.: «Las visitas de residencia como instrumento de control de la monarquía sobre el municipio foral: el caso de Alicante», en ALBALADEJO, P. (ed.), Monarquía, Imperio y Pueblos en la España Moderna. Actas de la IV Reunión Científica de la A.E.H.M., Vol. I, Alicante, 1997, pp. 561-568.

15. A.C.A., S.V., Leg. 679, Doc. 39.

16. A.C.A., S.V., Leg. 81 I, Doc. $40 / 39$. 
continuar este estilo por la dependencia del otro, pero que sirviéndole personalmente no se duda que sería a propósito y de conveniencia a la ciudad. ${ }^{17}$. Más contundente en su juicio se mostró el visitador D. Braulio Esteve, pese a que fue él quien le situó en el cargo; en carta de 18 de agosto de 1664, dirigida al vicecanciller del Consejo de Aragón, se expresaba en los siguientes términos: «Digo que sin género de duda Bautista Boyer, notario, a quien yo encomendé este officio, fuera el más a propósito si no se le inhabilitara por lo que he experimentado el tiempo que lo ha tenido, que jamás ha acudido a la Sala ni servidole sino por su sobrino Honorat Boyer», «la pereça de Bautista no tiene enmienda». De la otra candidatura que se barajaba, la de Pedro Maltés, Esteve también opina sin ambigüedades: «demás de ser caballero que me parece. sobrado estado para el officio a quien se havía de dar la autoridad de notario para recevir autos, no me pareçe al propósito su condición y es primo hermano de Joseph Arcayna y pareçe sería restituirle el officio por tal vía, siendo tan perjudicial» ${ }^{18}$.

Mientras se esperaba el fallo de la apelación suscitada por Arcayna, los justicia y jurados de la ciudad solicitaron y obtuvieron del rey el 10 de febrero de 1665 el nombramiento del Dr. Victoriano Tredós y Pascual como nuevo titular provisional de la escribanía. En principio, el nuevo escribano había sido elegido con el beneplácito de todos, tanto de las autoridades locales como de las reales, siendo el candidato preferido del virrey y del visitador Esteve, bajo cuyas órdenes había trabajado como abogado fiscal durante su visita de residencia ${ }^{19}$.

De los primeros años de ejercicio de Tredós no nos han Ilegado noticias que cuestionasen su labor al frente de la escribanía. Parecía, por fin, que se había acertado con su nombramiento. Sin embargo, algunos años después, concretamente en febrero de 1677 , Francisco Idiáquez, duque de Ciudad Real y virrey de Valencia, puso en conocimiento del Consejo de Aragón una denuncia anónima fechada en Alicante de la que reproducimos aquí los párrafos que más nos interesan:

«Mucho tiempo ha que se alla esta Ciudad oprimida en el mal Govierno de ella, perturbándole el Dr. Victoriano Tredos, escribano de los Jurados y Consejo, quexándose todo el pueblo de sus prosedimientos y mala administración que ay de las rentas y propios della, porque este sujeto, con su manía y disposición lo confunde todo, perturbando, con la mano que tiene, el que los jurados y officiales de la Ciudad no puedan obrar lo que deven por rasón de sus oficios que, como éstos son anuales, entran con pocas o ningunas noticias y no alcansan más que aquéllas que él les quiere participar y les çela las demás conveniencias de la Ciudad por las que a él se le siguen de ignorarlas, y no sólo causa este daño a las rentas de la Ciudad, sino también a muchos pobres maestros y officiales que trabaxan por la Ciudad en diferentes oficios, alargándoles las pagas y aún pasando a aprovecharse de muchas de ellas para suplir el excessivo gasto que tiene en su casa y familia, pues es tal que el de mayor lucimiento de la mejor casa y familia no le iguala, teniendo tan sólo para ello el officio, sin otro patrimonio ni rentas»" ${ }^{210}$.

A la denuncia dada a conocer por el virrey le seguiría una acusación formal por parte de dos de los jurados en ejercicio durante el año $1677^{21}$. La campaña de descré-

17. A.C.A., S.V., Leg. 805 , Doc. $40 / 5$.

18. A.C.A., S.V., Leg. 805. Doc. $40 / 7$.

19. A.C.A., S.V., Leg. 805 , Doc. $40 / 5$ y $40 / 7$.

20. A.C.A., S.V., Leg. 796 , Doc. 11.

21. A.C.A., S.V., Leg. 805, Doc. $40 / 26$. 
dito de Tredós había comenzado. Los prolegómenos de la misma ya nos son conocidos: envío a las autoridades reales de denuncias anónimas, inicio de acusaciones públicas por parte de ciertos oficiales municipales, llegada de un oficial designado por el rey para investigar los hechos... Lo que ya no está tan claro es por qué se tardó más de diez años en denunciar a Tredós si, como se desprende de la carta del virrey, los procedimientos irregulares del escribano ya eran de dominio público desde tiempo atrás. La consideración de que la renovación anual de la juraduría fuese un obstáculo para permitir a las primeras autoridades del municipio estar al tanto de los desmanes del escribano, tal y como se manifiesta en el anónimo llegado al virrey, es cuestionable y, desde luego, se presta a dos lecturas. Podríamos tomar esta apreciación como errónea, por cuanto que las acusaciones efectuadas por los jurados, como tendremos ocasión de comprobar, demuestran un seguimiento cercano de la contabilidad, lo cual nos llevaría a pensar que los anteriores mandatos, o bien habían actuado todos de forma negligente, o bien tenían «intereses» en la manera de proceder del escribano. Sin embargo, si la consideramos acertada, deberíamos preguntarnos si el celo demostrado por los jurados de 1677 obedecía a razones más profundas que la propia honestidad profesional. El hecho de que uno de los dos jurados firmantes fuera el ya conocido Dr. Pedro Maltés, pariente del escribano Arcayna, que aún reclamaba su puesto (el otro era Juan Bautista Canicia de Franquí ${ }^{22}$ ), ya nos hace sospechar que la consigna era, claramente, remover a Tredós del cargo.

Las acusaciones vertidas contra Tredós motivaron la apertura de un proceso que tendría unas consecuencias para el escribano excesivamente duras en relación al delito que se imputaba, consistente en una simple duplicidad en el despacho de unas órdenes de pago firmadas por los jurados para sufragar gastos de obras en conducciones de aguas, valorada en 315 libras. Una acusación rigurosa, por cuanto que no es infrecuente encontrar en los libros de contabilidad municipal registros de pago duplicados que, una vez detectados por el racional al cierre del balance, quedan regularizados, y una cifra ridícula si tenemos en cuenta el nivel presupuestario del municipio ${ }^{23}$.

Las actuaciones contra Tredós se iniciaron con su suspensión de oficio en junio de 1678. La inspección a que fue sometido fue encargada a D. Francisco Pastor, quien por aquellas fechas se encontraba en Orihuela ocupado en asuntos de prevención de un brote contagioso. Mientras tanto, sus funciones fueron temporalmente asumidas por el Dr. Mateo Rodrigo, a la sazón asesor de la Bailía, que se limitó a formalizar por escrito las denuncias de los jurados antes citados y a la recogida de nuevos testimonios. Cuando finalmente Pastor se personó en Alicante en diciembre de 1678 para iniciar el procedimiento, se encontró con la sorpresa de que parte de los testigos que habían testificado contra Tredós habían huido de la ciudad.

22. Juan Bautista Canicia coincidió con Joseph Arcayna en el gobierno municipal cuando aquél desempeñaba el oficio de síndico. Durante la visita de residencia de D. Braulio Esteve se le abrió proceso a Canicia por haber dilapidado en gastos personales casi 500 libras del presupuesto municipal en un viaje que realizó a Barcelona para proveer a la ciudad de armamento, según consta en el informe enviado a la corte por el propio visitador, fechado en Alicante el 8 de octubre de 1663. A.C.A., S.V., Leg. 679, Doc. 39.

23. Un informe del visitador Esteve cifraba las rentas de la ciudad de Alicante hacia 1665 en unas 33.000 libras. A.C.A., S.V., Leg. 679, Doc. 97. 
En un primer momento, el fiscal que asistía a Pastor en la causa, Dr. Francisco Bourgunyo, había decidido ampliar la instrucción contra Tredós, añadiendo un segundo cargo aparte del de la duplicación de partidas de gastos: el de obrar sin guardar los estatutos, aunque como bien reconocía el fiscal: «no sólo está comprendido el Dr. Tredós como secretario de esta ciudad, sí cassi todos los insaculados en su govierno que han servido sus mejores officios, como reos principales», concluyendo que, a este respecto, «no les allo confessados de oficio» ${ }^{24}$.

A finales de agosto del año siguiente, 1679, Pastor volvió a ausentarse dejando el proceso en manos de su abogado fiscal sin haber podido adelantar en la instrucción por las dificultades jurídicas que, al parecer, ocasionaba la actuación de los testigos: «los medios jurídicos para quitar semejantes embaraços son la questión del tormento y la accusación de falsedad contra los testigos. El de la tortura no se puede ajustar fácilmente en Pedro Valenzuela, Pedro Juan López y Antonio Penalva por estar ausentes, en Francisco Mora por ser ciudadano insaculado y en Joseph Pérez, septuagenario, por su edad y accidentes» ${ }^{25}$.

Por esas mismas fechas el rey había dispuesto que, una vez finalizase D. Francisco Pastor sus diligencias, se remitiera la causa al Consejo Supremo de Aragón para que éste dictase sentencia, permitiéndose a Tredós viajar personalmente a la Corte para hacer su propio alegato, en consideración a que su situación económica le impedía disponer de los servicios de un abogado procurador ${ }^{26}$.

Francisco Pastor, en el punto muerto en que se hallaba la causa, manifiesta en carta fechada en 27 de noviembre de 1679 sus dudas acerca de la necesidad de seguir. adelante. Era muy probable que, en caso de decidirse a abrir proceso contra los testigos bajo acusación de falsedad obtuviera la condena de los mismos. Era consciente, además, de que detrás de esta iniciativa se encontraba Joseph Arcayna pues mantenía litigio por su plaza. $Y$, por encima de todo, lo consideraba un proceso excesivamente oneroso, pues la duplicación de la orden de pago tramitada por Tredós, que efectivamente sí se produjo, no supuso mayor quebranto económico a la hacienda local que 35 libras, pues una vez revisados todos los apuntes contables realizados por Tredós no se detectaron otras irregularidades. Y sólo por eso, por un perjuicio de 35 libras, el acusado había sufrido un castigo desmedido durante dos años. Se le condenó a mantenerse alejado del municipio un mínimo de 20 leguas y, cuando regresó, a requerimiento de la ciudad, fue encarcelado durante ocho meses para, más tarde, recibir una orden de confinamiento en su domicilio hasta que concluyese el proceso ${ }^{27}$. Además, había que contar con los gastos ocasionados por la tramitación de la causa, valorada por Pastor en más de 500 ducados de plata, parte de los cuales se invirtieron en salarios y dietas de personal, incluidos sus ayudantes -dos alguaciles y un escribano"que he traído de fuera por no juzgar a propósito los de esta ciudad»"

Creemos que conviene, llegados a este punto, recapitular la complicada situación en la que se encontraba la plaza de escribano de la Sala. Tenemos, por una parte,

24. A.C.A., S.V., Leg. 805, Doc. $40 / 37$.

25. A.C.A., S.V., Leg. 805 , Doc. $40 / 26$

26. A.C.A., S.V., Leg. 805 , Doc. $40 / 33$.

27. A.C.A., S.V., Leg. 805 , Doc. $40 / 10$ y $40 / 36$.

28. A.C.A., S.V., Leg. 805 , Doc. $40 / 26$. 
a Joseph Arcayna, suspendido por proceso abierto en su contra cuya apelación aún estaba pendiente de decidir y, por otra, a Victoriano Tredós, sustituto del anterior, de forma se entiende que provisional, también suspendido por otro proceso. Antes de que se conociera el fallo de su apelación falleció Joseph Arcayna. En ese preciso momento se produjo una vacante real, por muerte del titular y suspensión del escribano provisional. La ciudad decidió entonces designar como escribano al notario Gínés Gosálbez. Y todo se complicó aún más. Como consecuencia de este nombramiento y de la dilación que sufría su causa, Victoriano Tredós impugnó ante la Real Audiencia de Valencia la elección de Gosálbez. En contrapartida, la ciudad entabló pleito contra Tredós, amparándose en el acta de las Cortes de 1645 que les concedía la facultad de nombrar al titular ${ }^{20}$.

La Real Audiencia resolvería finalmente el litigio a favor de Tredós, declarando nulo el nombramiento de Gosálbez. No contenta con el fallo, la ciudad recurrió en la misma Audiencia la resolución, solicitando al Consejo de Aragón que la causa fuese remitida ante la última instancia ${ }^{30}$. Pero la última tentativa del gobierno municipal por apartar a Tredós del Consell local resultaría inútil. Finalmente, el Consejo falló en favor de Victoriano Tredós y en julio de 1680 una real orden ordenaba al municipio la aceptación del dictamen y la restitución del escribano en su oficio ${ }^{31}$.

La reincorporación de Victoriano Tredós al gobierno municipal no estuvo exenta de impedimentos. No debió ser fácil enfrentarse con aquéllos que le habían denunciado aunque, evidentemente, Tredós, como miembro de la oligarquía local y sujeto activo de una de sus facciones, dispuso de importantes respaldos que le aseguraron la permanencia en su oficio ${ }^{32}$.

Las vicisitudes por las que atravesó la provisión del oficio de escribano de la Sala del Consell que aquí hemos recogido constituyen una buena muestra de los principios que regían la práctica política de estos oficiales de gobierno. Pero, desde luego, no es única. Los distintos oficiales reales que inspeccionaron la administración alicantina a lo largo del siglo XVII rara vez se toparon con un oficial libre de sospecha. Creemos que puede decirse, casi con total seguridad, que todos y cada uno de los miembros del Consell municipal esperaban su turno en la alternancia del gobierno ciudadano para preservar sus propios intereses y los de su «clientela», y que esta lucha por el poder se desarrollaba en un ambiente en el que las delaciones y conspiraciones eran considerados como mera cotidianidad. Sirva como ejemplo el contenido de una nueva denuncia anónima que llegó hasta el Consejo de Aragón dos años después del regreso de Tredós:

«toda la gente perdida, viziada y despresiable se an puesto del vando maior, de suerte que los de la primera sangre, nobleza y caudal se han abstraído de los cargos y ofizios propios desde que volvió el Dr. Victoriano Tredós a la secretaría de la Ciudad, con que su herario, caudal y govierno a quedado únicamente a la discrección de los que llevo di-

29. A.C.A., S.V., Leg. 805, Doc. $40 / 13$.

30. Ibídem.

31. A.C.A., S.V., Leg. 805 , Doc. $40 / 16$.

32. Desconocemos la fecha exacta en que dejó vacante la escribanía Victoriano Tredós, pero podemos confirmar que en 1699 seguía al frente de la misma, según demucstra la documentación contable del municipio correspondiente a ese año. 
cho y, aunque la fantasma de la visita de D. Francisco Pascual ${ }^{33}$ sólo era un freno que detendrá algunos precipizios, biéndose éste en el retiro e ignorancia afectado de todo, obran más confiados en lo no devidos ${ }^{34}$.

33. D. Francisco Pascual ejerció como visitador tras la muerte de su padre, D. Alejandro Pascual de lbarra, quien hacia noviembre de 1665 había sustituido a D. Braulio Esteve. D. Francisco se mantuvo en sus funciones hasta que fue cesado el 8 de agosto de 1683. A.C.A., S.V., Leg. 621, Doc. 13.

34. A.C.A., S.V., Leg. 815 , Doc. $32 / 3$. 